\title{
Image Compression by Discrete Curvelet Wrapping Technique with Simplified SPHIT
}

\author{
K. Siva Nagi Reddy \\ Associate professor, Dept of ECE, \\ MSSIST-College of Engineering, \\ Mylavaram, Vijayawada, \\ Krishna (D.t), Andhra- Pradesh, India.
}

\author{
B. R.Vikram \\ Professor, Dept of ECE, \\ Vijay Rural Engineering College, \\ Nizamabad, Nizamabad (D.t), \\ Andhra- Pradesh, India.
}

\begin{abstract}
The proposed paper is to develop an efficient compression scheme and to obtain better quality and higher compression ratio through discrete curvelet transform and embedded coding of curvelet coefficients through improved Set Partitioning In Hierarchical Trees algorithm (SPIHT) algorithm. The paper demonstrates a significant improvement in visual quality and faster encoding and decoding than the wavelet with SPHIT compression. The SPHIT with wavelet compression fail to represents discontinuous along the curves. The curvelet transform is a multiscale directional transform, which allows an almost optimal non adaptive sparse representation of objects with edges. By using improved SPHIT with Curvelets model the transform coefficients based on probability of significance, at a fixed threshold of the offspring. As far as objective quality assessment of the image compression of the proposed work will gives improved Peak Signal to Noise Ratio (PSNR) and high compression ratio (CR) compared with the existing wavelet transform with SPHIT image compression.
\end{abstract}

\section{Keywords}

curvelet, SPHIT, DCT, Subband, Compression, DWT, FFT.

\section{INTRODUCTION}

Image compression is used to reduce the image data as small as possible under an acceptable limit of errors. In general, the image compression techniques can be classified into two major categories: loss and lossless. Lossy compression provides less disk space, but also the transmission time or bandwidth is concerned while lossless compression can completely reconstruct the original data. In addition, lossless data compression offers perfect data transmission. Till now we are seen many advanced image-compression techniques have been developed in response to the ever-increasing demands for business and medical imaging [1]. EZW and SPIHT [2], [3] methods were offer hierarchical decomposition and utilize space or frequency localization of subband image data for energy compaction. JPEG2000 [4],[5] combines embedded block

\author{
L. Koteswara Rao \\ Asst. Professor, Dept of ECE, \\ Faculty of Science \& Technology, \\ IFHE (University), Hyderabad, \\ Andhra- Pradesh, India.
}

\author{
P. Ravikanth \\ Professor, Dept of ECE, \\ Sridevi woman's Engineering College \\ V.N.pally, Hyderabad, \\ Andhra- Pradesh, India.
}

coding with optimized truncation(EBCOT) technique with lifting integer wavelet transform to achieve highperformance image compression. Modified SPIHT schemes [6], [7] have also received considerable attention in the lossless image compression. However, the compression will reduce the image fidelity, especially when the images are compressed at lower bit rates. The reconstructed images suffer from blocking artifacts and the quality of the image is severely degraded .In recent years, much of the research activities in image coding have been focused on the discrete wavelet transform (DWT) as the overlapping nature of the transform alleviates blocking artifacts, while the multi-resolution character of the wavelet decomposition leads to grate energy compaction and good quality of the decompressed image. Furthermore, the multi-resolution transform domain means that wavelet compression methods degrade much more gracefully than block-DCT methods as the compression ratio increases. As a result, a new International Standard for image coding called JPEG2000. This introduction has meant that for the first time, the discrete wavelet transform is to be used for the decomposition and reconstruction of images together with an efficient coding scheme. In the wavelet transform there is an inability to represent edge discontinuities along the curves. Due to the large or several coefficients are used to reconstruct edges properly along the curves. For this reason, it needs a transform to handle the two dimensional singularities along the sparsely curve. This is the reason behind the birth of Curvelet transform. Here the Curvelet basis elements have wavelet basis and the edge discontinuities and other singularities better than the wavelet transform. So for the discrete curvelet transform, modifications must be made in the normal SPIHT Algorithm to get the efficient image compression.

In this paper, we proposed a new scheme which provides improvement in the quality of the compression image in terms of PSNR by Fast Discrete Curvelet transform [8] [9] with SPIHT simplified the original algorithm in [10] and modeled the transformed coefficient according to its significance in a $2 \times 2$ adjacent offspring group. 
The rest of the paper is organized as follows: section 2: Discuss the Methodology (Curvelet Transform introduction, types and algorithm) section 3.Reviews the SPIHT coding briefly and Section 4.discuss the Simplified SPHIT for curvelet image compression and section 5 . Discus the Simulation result and comparisons with wavelet transform.

\section{METHODOLOGY}

\subsection{Introduction of Curvelets}

The wavelets is mainly due to the best performance for piecewise smooth functions in 1-D.and the wavelets suffers mainly with 2-D line singularities this can be solved by first generation of the curvelets these are called ridgelets but those are used rarely in real time applications. Curvelets were introduced in [11] to address the edge representation problem is based on windowed ridgelets. In 2002 , they simplified the definition of curvelets and constructed anew tight frame. In [12], they developed a Continuous Curvelet Transform has been shown to be a very efficient tool for many different applications in image processing, data exploration in remote sensing, and image denoising.Curvelets will be superior over wavelets in optimally sparse representation of objects with edges, wave propagation, and mainly do better image reconstruction in severely ill posed problem of images. The curvelets offer optimal sparseness for "curve punctuated smooth" images, where the image is smooth with the exception of discontinuities along $\mathrm{C}^{2}$ curves. Sparseness is measured by the rate of decay of the n-term approximation (reconstruction of the image using $n$ number of coefficients) of the algorithm. The sparse representation offering to us like improved compression, denoising and increases the amount of smooth areas in the image. In [13] it was shown that orthogonal systems have optimal $\mathrm{n}$-term approximations that decay in $\mathrm{L}^{2}$ with rate $\mathrm{O}$ $\left(\mathrm{n}^{-2}\right)$ (as a lower bound). On images with $\mathrm{C}^{2}$ boundaries, non-optimal systems have the rates:

Fourier Approximation:

$\left\|\mathrm{f}-\mathrm{f}_{\mathrm{n}}^{\mathrm{F}}\right\|^{2} \mathrm{~L}^{2}=\mathrm{O}\left(\mathrm{n}^{\frac{-1}{2}}\right)$

Wavelet Approximation:

$\left\|\mathrm{f}-\mathrm{f}_{\mathrm{n}}^{\mathrm{w}}\right\|^{2} \mathrm{~L}^{2}=\mathrm{O}\left(\mathrm{n}^{-1}\right)$

Curvelet Approximation:

$\left\|\mathrm{f}-\mathrm{f}_{\mathrm{n}}^{\mathrm{C}}\right\|^{2} \mathrm{~L}^{2}=\mathrm{O}\left((\log \mathrm{n})^{3}\left(\mathrm{n}^{-2}\right)\right)$

As seen from the $n$-term approximations, the Curvelet Transform offers the closest n-term approximation to the lower bound. Therefore, in images with a large number of $\mathrm{C}^{2}$ curves (i.e. an image with a great number of long edges), it would be advantageous to use the Curvelet Algorithm.
Continuous Curvelet Transform (CCT): The Continuous Curvelet Transform [11] used a complex series of steps involving the ridgelet analysis of the radon transform of an image. Performance was exceedingly slow. The CCT is related to a continuous transform which is based on true affine parabolic scaling of a single mother wavelet, while the CCT can viewed as true affine parabolic scaling in Euclidean coordinates by multiple mother wavelet at each scale. CCT provide a continuous tight frame. In the CCT, the curvelet frame elements are not parabolic dilations all of a single generating function; there is a little variation in the generating function from one scale to the next scale. In the continuous case, the wavelets involved in the discrete tight frame are very close to affine scaling of a single mother wavelet. The algorithm was modified in 2003 in [12]. The use of the Ridgelet Transform was discarded, thus reducing the amount of redundancy in the transform and increasing the speed considerably.

Discrete Curvelet Transform (DCT): Using the theoretical basis in DCT there are two separate algorithms is introduced in [14]. The first algorithm is the non equispaced FFT Transform [15], by using that the curvelet coefficients of images are found by irregularly sampling the Fourier coefficients. The second algorithm is the Wrapping transform, by using that a series of translations and a wraparound technique. Both algorithms having the same output, but the Wrapping Algorithm gives us faster computation time. Basically, multi resolution discrete curvelet transform in the spectral domain utilizes the advantages of fast Fourier transform (FFT). During FFT, both the image and the curvelet at a given scale and orientation are transformed into the Fourier domain. The convolution of the curvelet with the image in the spatial domain then becomes their product in the Fourier domain. At the end of this computation process, we obtain a set of curvelet coefficients by applying inverse FFT to the spectral product. This set contains curvelet coefficients in ascending order of the scales and orientations. There is a problem in applying inverse FFT on the obtained frequency spectrum. The frequency response of a curvelet is a trapezoidal wedge which needs to be wrapped into a rectangular support to perform the inverse Fourier transform. The wrapping of this trapezoidal wedge is done by periodically tiling the spectrum inside the wedge and then collecting the rectangular coefficient area in the origin. Through this periodic tiling, the rectangular region collects the wedge's corresponding fragmented portions from the surrounding parallelograms. For this wedge wrapping process, this approach of curvelet transform is known as the wrapping based curvelet transform. 


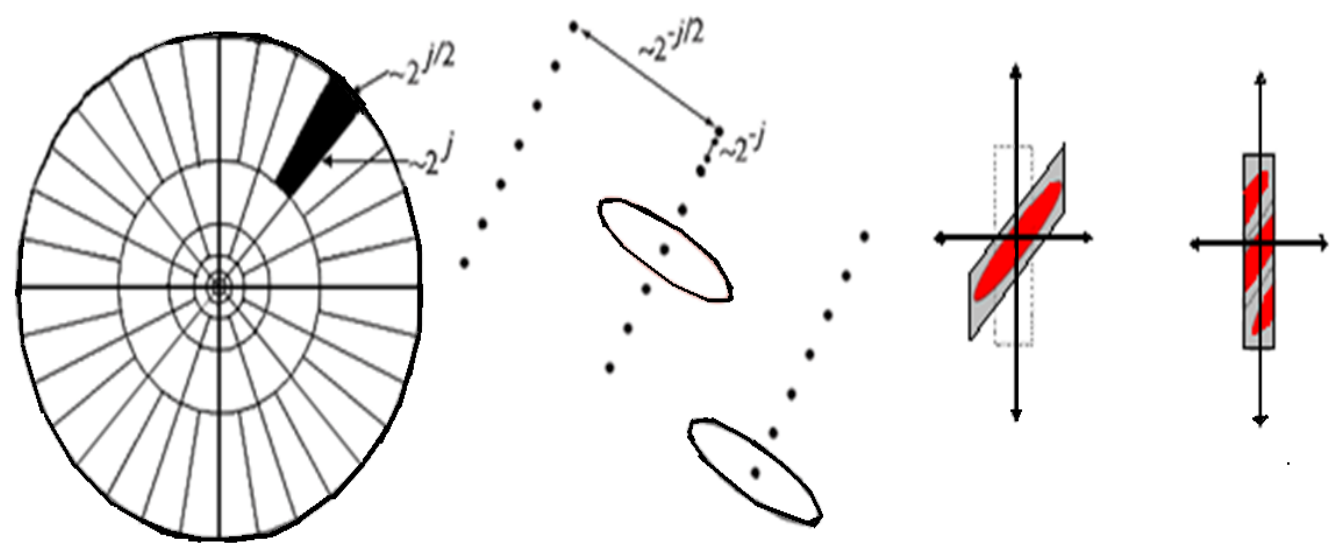

Figure.1: .a) Digital Corona of the Frequency Domain b) Support of Wedge before wrapping c) Support of Wedge after Wrapping

\subsection{Discrete Curvelet Transform}

Let the equidistant sequences of rotation angle $\theta_{\mathrm{j}, \mathrm{l}}:=$ $\frac{\pi / 2^{-[j / 2]}}{2}$ with $1=0,1 \ldots 4$.

$2^{[\mathrm{j} / 2]}-1$; Let the positions $\mathrm{b}_{\mathrm{k}}^{\mathrm{j}, \mathrm{l}}=\mathrm{b}_{\mathrm{k} 1, \mathrm{k} 2}^{\mathrm{j}, \mathrm{l}}:=\mathrm{R}_{\theta_{\mathrm{j}, 1}}^{-1}\left(\frac{\mathrm{k}_{1}}{2^{\mathrm{j}}}, \frac{\mathrm{k}_{2}}{2^{\frac{\mathrm{j}}{2}}}\right)^{\mathrm{T}}$, with $\mathrm{k}_{1}, \mathrm{k}_{2} \in \mathbb{Z}$, where $\quad \mathrm{R}_{\boldsymbol{\theta}}$ denotes the rotation matrix with angle $\boldsymbol{\theta}$ length $\approx 2^{-\frac{\mathrm{j}}{2}}$ and width $\approx 2^{-j}$ the Cartesian window in polar coordinates constructed as $\mathrm{U}_{\mathrm{j}}(\mathrm{r}, \mathrm{w})=2^{-3 \mathrm{j} / 4} \mathrm{~W}\left(2^{-\mathrm{j}} \mathrm{r}\right) \mathrm{V}\left(\frac{2.2^{[\mathrm{j} / 2]} \mathrm{w}}{\pi}\right)=$ $2^{-3 \mathrm{j} / 4} \mathrm{~W}\left(2^{-\mathrm{j}} \mathrm{r}\right) \mathrm{V}\left(\frac{\mathrm{w}}{\theta_{\mathrm{j}, \mathrm{l}}}\right), \mathrm{j} \in \mathbb{N}_{0}$

The basic curvelet is defined by $\widetilde{\phi}_{\mathrm{j}, 0,0}(\xi):=\mathrm{U}_{\mathrm{j}}(\xi)$,and the curvelet functions is given by

$\phi_{\mathrm{j}, \mathrm{k}, \mathrm{l}}(\mathrm{x})=\phi_{\mathrm{j}, 0,0}\left(\mathrm{R}_{\mathrm{\theta}_{\mathrm{j}, 1}}\left(\mathrm{x}-\mathrm{b}_{\mathrm{k}}^{\mathrm{j}, \mathrm{l}}\right)\right)$

The curvelet function in frequency domain given by $\widetilde{\phi}_{\mathrm{j}, \mathrm{k}, \mathrm{l}}(\xi)=\mathrm{e}^{-\mathrm{i}\left(\mathrm{b}_{\mathrm{k}}^{\mathrm{j}, \mathrm{l}}, \xi\right)} \mathrm{U}_{\mathrm{j}}\left(\mathrm{R}_{\theta_{\mathrm{j}, 1}} \xi\right)=$ $e^{-i\left(b_{k}^{j,}, \xi\right)} 2^{-3 j / 4} W\left(2^{-j} r\right) V\left(\frac{w+\theta_{j .1}}{\theta_{j .1}}\right)$

The discrete curvelet transform for every function $f \in \mathrm{L}^{2}\left(\mathbb{R}^{2}\right)$ is given as invertible. $f=\sum_{\mathrm{j}, \mathrm{k}, \mathrm{l}}\left\langle\mathrm{f}, \phi_{\mathrm{j}, \mathrm{k}, \mathrm{l}}\right\rangle \phi_{\mathrm{j}, \mathrm{k}, \mathrm{l}}$ (7)

The terms $c_{\mathrm{j}, \mathrm{k}, \mathrm{l}}(\mathrm{f}):=\left\langle\mathrm{f}, \phi_{\mathrm{j}, \mathrm{k}, \mathrm{l}}\right\rangle$ are called curvelet coefficients.

$c_{j, k, l}(f)=\int f(x) \overline{\phi_{j, k, l}(x)} d x=\int \hat{f}(\xi) \overline{\widetilde{\phi}_{j, k, l}(\xi)} d \xi=$

$\int \hat{\mathrm{f}}(\xi) \mathrm{U}_{\mathrm{j}}\left(\mathrm{R}_{\theta_{\mathrm{j}, 1}} \xi\right) \mathrm{d} \xi=\mathrm{e}^{\mathrm{i}\left(\mathrm{b}_{\mathrm{k}}^{\mathrm{j}, 1}, \xi\right)} \mathrm{d} \xi$

(8)

Replace the window $W_{j}(r):=W\left(2^{-j} r\right)$

Then the Radial Window form

$\widetilde{W}_{j}(r)=X_{[0, \infty]}(r) \cdot \sqrt{r^{2}\left(2^{-j-1} r\right)-\gamma^{2}\left(2^{-j} r\right)} . \quad j \geq 0, r \geq$ 0 .

Hence suppose $\widetilde{W_{j}}=\left[2^{j-1}, 2^{j+1}\right]$.

The angular window given as $\mathrm{V}_{\mathrm{j}}(\xi)=\mathrm{V}\left(2^{[\mathrm{j} / 2]} \xi_{2} / \xi_{1}\right)$

Now from (4) the Cartesian window, $\widetilde{\mathrm{U}}_{\mathrm{j}}(\xi)=$

$\left.2^{-3 \mathrm{j} / 4} \widetilde{\mathrm{W}}_{\mathrm{j}}\left(\xi_{1}\right) \mathrm{V}_{\mathrm{j}}(\xi)=2^{-3 \mathrm{j} / 4} \widetilde{\mathrm{W}}_{\mathrm{j}} \xi_{1}\right) \mathrm{V}_{\mathrm{j}}\left(\frac{2^{[\mathrm{j} / 2]} \xi_{2}}{\xi_{1}}\right)$

The Cartesian window $\mathrm{U}_{\mathrm{j}}$ in (4) and
The frequencies in the trapezoid Tan $\theta_{\mathrm{j}, \mathrm{t}}:=12^{-[\mathrm{j} / 2]}$, $\mathrm{l}=-2^{[\mathrm{j} / 2]}+1 \ldots \ldots, 2^{[\mathrm{j} / 2]}-1$. the value of $\theta_{\mathrm{j}, \mathrm{l}}=$ $-\pi / 4$ to $\pi / 4$

Now the curvelet counter function is given by $\widehat{\widetilde{\phi}}_{\mathrm{j}, \mathrm{k}, \mathrm{l}}(\mathrm{x})=$ $\widetilde{\mathrm{U}}_{\mathrm{j}}(\xi), \widetilde{\phi}_{\mathrm{j}, \mathrm{k}, \mathrm{l}}(x)=$

$\widetilde{\phi}_{\mathrm{j}, 0,0}\left(\mathrm{~S}_{\widetilde{\phi}_{\mathrm{j}, 1}^{\mathrm{T}}}^{\mathrm{T}}\left(\mathrm{x}-\tilde{\mathrm{b}}_{\mathrm{k}}^{\mathrm{j}, \mathrm{l}}\right)\right.$

The Cartesian counterpart of $\widehat{\widetilde{\phi}}_{\mathrm{j}, \mathrm{k}, \mathrm{l}}$ in (6), with the shear matrix $S_{\theta}=\left(\begin{array}{cc}1 & 0 \\ -\tan \theta & 1\end{array}\right)$,

And where $\widetilde{b}_{\mathrm{k}}^{\mathrm{j}, \mathrm{l}}=\mathrm{S}_{\theta_{\mathrm{j}, 1}}^{-\mathrm{T}}\left(\mathrm{k}_{1} 2^{-\mathrm{j}} \mathrm{k}_{2} 2^{-[\mathrm{j} / 2]}\right)=\mathrm{S}_{\theta_{\mathrm{j}, 1}}^{-\mathrm{T}} \mathrm{k}_{\mathrm{j}} \quad$ The Fourier Transform given by $S_{\theta_{j, 1}}^{-1} \xi=\left(\xi_{1}, \xi_{1} \tan \theta_{j, 1}+\xi_{2}\right)^{\mathrm{T}}$

Now finally the curvelet function by $\widehat{\widehat{\phi}}_{\mathrm{j}, \mathrm{k}, \mathrm{l}}(\xi)=$ $\mathrm{e}^{-\mathrm{i}\left(\widetilde{\mathrm{b}}_{\mathrm{k}}^{\mathrm{j}, 1}, \xi\right)} \widehat{\widetilde{\phi}}_{\mathrm{j}, 0,0}\left(\mathrm{~S}_{\theta_{\mathrm{j}, 1}^{-1}} \xi\right)=\mathrm{e}^{-\mathrm{i}\left(\widetilde{\mathrm{b}}_{\mathrm{k}}^{\mathrm{j}, 1}, \xi\right)} \widetilde{\mathrm{U}}_{\mathrm{j}}\left(\mathrm{S}_{\theta_{\mathrm{j}, 1}}^{-1} \xi\right)=$

$\mathrm{e}^{-\mathrm{i}\left(\widetilde{\mathrm{b}}_{\mathrm{k}}^{\mathrm{j}, 1}, \xi\right)} 2^{-3 \mathrm{j} / 4} \widetilde{\mathrm{W}}_{\mathrm{j}}\left(\xi_{1}\right) \mathrm{V}_{\mathrm{j}}\left(\mathrm{S}_{\theta_{\mathrm{j}, 1}}^{-1} \xi\right)=\mathrm{e}^{-\mathrm{i}\left(\tilde{\mathrm{b}}_{\mathrm{k}}^{\mathrm{j}, 1}, \xi\right)} 2^{-3 \mathrm{j} / 4} \widetilde{\mathrm{W}}_{\mathrm{j}}\left(\xi_{1}\right)$

$\mathrm{V}\left(2^{[\mathrm{j} / 2]} \xi_{2} / \xi_{1}+\mathrm{l}\right)$.

From (12) and (7) in equation (8) Then curvelet coefficients is given by $\tilde{\mathrm{c}}_{\mathrm{j}, \mathrm{k}, \mathrm{l}}(f)=\left\langle f, \widetilde{\phi}_{\mathrm{j}, \mathrm{k}, \mathrm{l}}\right\rangle$ $=\int \hat{\mathrm{f}}(\xi) \overline{\mathrm{U}}_{\mathrm{j}}\left(\mathrm{S}_{\theta_{\mathrm{j}, 1}}^{-1} \xi\right) \mathrm{e}^{-1\left(\widetilde{\mathrm{b}}_{\mathrm{k}}^{\mathrm{j}, 1}, \xi\right)} \mathrm{d} \xi=$ $\int \tilde{\mathrm{f}}\left(\mathrm{S}_{\theta_{\mathrm{j}, 1}} \xi\right) \overline{\mathrm{U}}_{\mathrm{j}}(\xi) \mathrm{e}^{\mathrm{i}\left(\mathrm{k}_{\mathrm{j}}, \xi\right)} \mathrm{d} \xi$

(13) withk $_{\mathrm{j}}=$ $\left(\mathrm{k}_{1} 2^{-\mathrm{j}}, \mathrm{k}_{2} 2^{-[\mathrm{j} / 2]}\right)^{\mathrm{T}},\left(\mathrm{k}_{1}, \mathrm{k}_{2}\right)^{\mathrm{T}} \in \mathbb{Z}^{2}$

\subsection{Algorithm}

1. Apply the Fourier transform of $f$ by means of a 2D FFT. Let $\mathrm{f}$ be given by its samples $\mathrm{f}\left(\mathrm{n}_{1} / \mathrm{N}, \mathrm{n}_{2} / \mathrm{N}\right), \mathrm{n}_{1}, \mathrm{n}_{2}=0, \ldots \ldots \ldots \mathrm{N}$ 1 , Where $\mathrm{N}=2^{\mathrm{j}}$, JEN. $\mathrm{f}$ is approximated by linear combination of bi-variate hat function. Let $\tilde{s}(\mathrm{x})=$ $\mathrm{s}\left(\mathrm{x}_{1}\right) \mathrm{s}\left(\mathrm{x}_{2}\right)$ with $\mathrm{s}\left(\mathrm{x}_{1}\right)=$

$\left(1-\left|\mathrm{x}_{1}\right| \mathrm{X}[-1,1]\left(\mathrm{x}_{1}\right) \quad \& \mathrm{f}(\mathrm{x})=\right.$

$\sum_{\mathrm{n} 1=0}^{\mathrm{N}-1} \sum_{\mathrm{n} 2=0}^{\mathrm{N}-1} \mathrm{f}\left(\frac{\mathrm{n}_{1}}{\mathrm{~N}}, \frac{\mathrm{n}_{2}}{\mathrm{~N}}\right) \tilde{\mathrm{s}}\left(\mathrm{Nx}_{1}-\mathrm{n}_{1}, \mathrm{Nx}_{2}-\mathrm{n}_{2}\right)$

(14)

with $\widehat{\widetilde{s}}(\xi)=\left(\xi_{1} / 2\right)^{2}\left(\xi_{2} / 2\right)^{2}, \hat{\mathrm{f}}(\xi)=$

$\sum_{\mathrm{n} 1=0}^{\mathrm{N}-1} \sum_{\mathrm{n} 2=0}^{\mathrm{N}-1} \mathrm{f}\left(\frac{\mathrm{n}_{1}}{\mathrm{~N}}, \frac{\mathrm{n}_{2}}{\mathrm{~N}}\right) \mathrm{e}^{\frac{-\mathrm{i}\left(\mathrm{n}_{1} \xi_{1}+\mathrm{n}_{2} \xi_{2}\right)}{\mathrm{N}}} \widetilde{\mathrm{s}}\left(\frac{\xi}{\mathrm{N}}\right)$

The 2D FFT of length $\mathrm{N}$ gives the samples $\hat{\mathrm{f}}\left(2 \pi \mathrm{n}_{1}, 2 \pi \mathrm{n}_{2}\right), \mathrm{n}_{1}, \mathrm{n}_{2}=\frac{-\mathrm{N}}{2}, \ldots . \frac{-\mathrm{N}}{2}-1$ 
2. Apply $\hat{\mathrm{f}}\left(\mathrm{S}_{\theta_{\mathrm{j}, \mathrm{l}}} \xi\right)$ by interpolation the scale is $\mathrm{j}_{0} \leq \mathrm{J} \leq \mathrm{J}$. the $\widetilde{\mathrm{U}}_{\mathrm{j}}$ is in rectangle

$R_{j}=\left[\left[2^{J-1}, 2^{J+1}\right] \times\left[-2^{\left|\frac{1}{2}\right|}, 2^{\left|\frac{\mid}{2}\right|},\right] \quad\right.$ (16) Each pair $(j / l)$ apply now $\hat{\mathrm{F}}\left(2 \pi \mathrm{n}_{1}, 2 \pi \mathrm{n}_{2}-2 \pi \mathrm{n}_{1} \tan \theta_{\mathrm{j}, \mathrm{l}}\right)$ for $2 \pi\left(\mathrm{n}_{1}, \mathrm{n}_{2}\right) \in$ $\mathrm{R}_{\mathrm{j}}$

3. Wrap the product around the origin and obtain $\hat{\mathrm{f}}\left(\mathrm{S}_{\theta_{\mathrm{j}}, 1} \xi\right) \widetilde{\mathrm{U}}_{\mathrm{j}}(\xi)$ for each pair $(\mathrm{j}, 1)$ computes the product $\widehat{\mathrm{f}}\left(2 \pi \mathrm{n}_{1}, 2 \pi \mathrm{n}_{2}-2 \pi \mathrm{n}_{1} \tan \theta_{\mathrm{j}, 1}\right) \widetilde{\mathrm{U}}_{\mathrm{j}}\left(2 \pi \mathrm{n}_{1}, 2 \pi \mathrm{n}_{2}\right)$

(17)

4. Apply the inverse 2D FFT in order to get the discrete coefficients $c_{j, k, l}^{D}(f)$ that are an approximation of the coefficients in equation (13)

\section{SPIHT ALGORITHIM}

In [16] the SPIHT algorithm, the image is first decomposed into a number of subbands. The subband coefficients are then grouped into sets known as spatialorientation trees, which efficiently exploit the correlation between the frequency bands. The coefficients in each spatial orientation tree are then progressively coded from the most significant bit-planes (MSB) to the least significant bit-planes (LSB), starting with the coefficients with the highest magnitude and at the lowest pyramid levels. The SPIHT multistage encoding process employs three lists and sets:

1. The list of insignificant pixels (LIP) contains individual coefficients that have magnitudes smaller than the threshold.

2. The list of insignificant sets (LIS) contains sets of wavelet coefficients that are defined by tree structures and are found to have magnitudes smaller than the threshold (insignificant).The sets exclude the coefficients corresponding to the tree and all sub-tree roots and they have at least four elements.

3. The list of significant pixels (LSP) is a list of pixels found to have magnitudes larger than the thresh-old (significant).

4. The set of offspring (direct descendants) of a tree node, $\mathrm{O}(\mathrm{i}, \mathrm{j})$, in the tree structures is defined by pixel location ( $\mathrm{i}$, $j)$. The set of descendants $D(i, j)$, of a node is defined by pixel location $(i, j)$. $O(i, j)$ is defined as $L(i, j)=D(i, j)$ $-\mathrm{O}(\mathrm{i}, \mathrm{j})$.The threshold, $\mathrm{T}$, for the first bit-plane is equal to $2^{\mathrm{n}}$ and

$\mathrm{n}=\log _{2}\left(\max \left\{\left|\mathrm{c}_{(\mathrm{i}, \mathrm{j})}\right|\right\}\right.$

Where $c(i, j)$ represents the $(i, j){ }^{\text {th }}$ transform coefficient. For operations in the subsequent bit-planes of threshold $\mathrm{T}$, $\mathrm{n}$ is reduced by 1 . Where $\mathrm{c}(\mathrm{i}, \mathrm{j})$ represents the $(i, j)$ th transform coefficient.

For each pixel in the LIP, one bit is used to de-scribe its significance. If it is not significant, the pixel remains in the LIP and no more bits are generated; otherwise, a sign bit is produced and the pixel is moved to the LSP. Similarly, each set in the LIS requires one bit for the significance information. The insignificant sets remain in the LIS; the significant sets are partitioned into subsets, which are processed in the same manner and at the same resolution until each significant subset has exactly one coefficient. Finally, each pixel in the LSP is refined with one bit. The abovementioned procedure is then repeated for the subsequent resolution. The algorithm has several advantages. The first one is an intensive progressive capability -the decoding (or coding) can be interrupted at any time and a result of maximum possible detail can be reconstructed with one-bit precision. This is very desirable when transmitting files over the internet, since users with slower connection speeds can download only a small part of the file, obtaining much more usable result when compared to other codec such as progressive JPEG. Second advantage is a very compact output bit stream with large bit variability - no additional entropy coding or scrambling has to be applied.

But SPIHT coding also have some disadvantages. SPIHT is very vulnerable to bit corruption, as a single bit error can introduce significant image distortion depending of its location. Much worse property is the need of precise bit synchronization, because a leak in bit transmission can lead to complete misinterpretation from the side of the decoder. For SPIHT to be employed in real-time applications, error handling and synchronization methods must be introduced in order to make the codec more resilient.

\section{SIMPLIFIED SPIHT METHOD FOR CURVELET}

In order to simplify the SPIHT coder [17] suggest two concepts for the SPIHT coding procedures.

First Modification: In the SPIHT coding process, a lesser number of bit-planes are discarded for higher target bitrates. Inversely, more bit-planes are discarded for lower target bit-rates. If we can determine the relationship between the bit-planes and target bit-rates, immediately discard the number of bit-planes to get different target bitrates.

Second Modification: at low bit-rates (implying that some bit-planes are to be discarded), if a subband coefficient is slightly lower than $2^{\mathrm{n}}$ and considerably larger than $2^{(\mathrm{n}-1)}$, we should then regard the coefficient as being "significant," and one bit is used to describe its significance. It is apparent that the other LSB bit-planes are not coded and the compression rate is improved.

\subsection{Two Modifications for Simplified SPIHT Algorithm}

First, the refinement pass in the original SPIHT is discarded. Therefore, if the curvelet coefficient is greater than or equal to the threshold $\mathrm{T}$, it is directly coded into the bit-stream and the coordinate node is immediately discarded from the data structure list. In this paper, we adaptively select all the bits at a time, while the curvelet coefficient is decided on the basis of significance. The direct coding of all the bits of significant coefficients does not greatly deteriorate the coding performance. In order to identify the required number of encoded bits, we should explore the statistical inference between the bit-rates and the number of bit-planes. It is apparent that different target bit-rates should correspond to different discarded bitplanes. Therefore, for different target bit-rates $\left(\mathrm{R}_{\mathrm{tb}}\right)$, expressed in bits per pixel (bpp), by using a simple decision rule defined as 
$\mathrm{N}_{\mathrm{db}}= \begin{cases}{\left[-\log \mathrm{R}_{\mathrm{tb}}\right]+\mathrm{t}, \mathrm{R}_{\mathrm{tb}} \leq 1} \\ 0 & \text { other wise }\end{cases}$

Where $\mathrm{N}_{\mathrm{db}}$ represents the number of discarded bit-planes in the order from the LSB to the MSB; $t$ denotes the quality control parameter. For the most part, the acceptable and normal images have the same T. It is apparent that the above modification continues to maintain a similar embedded property behavior in the SPIHT bitstream. By the above modification, quantization and refinement steps in the entire coding procedure can be avoided.

The second modification, combined with the first modification, is used to improve the image quality by using the adaptive threshold $\mathrm{T}$. If the curvelet coefficient is classified as "insignificant" based on $\mathrm{T}$, it is not discarded immediately. If the coefficient is very close to $\mathrm{T}$, it may be reclassified as being "significant." Therefore, the adaptive threshold is expressed as $T_{a}=(T-f)$. Where $f$ is an adaptive quantization parameter for the best image compression performance by statistical analyses of the test images, which varies with the target bit-rate as defined by

$$
\mathrm{f}=\left\{\begin{array}{lr}
0, & 1<\mathrm{R}_{\mathrm{tb}} \\
2, & 0.75<\mathrm{R}_{\mathrm{tb}} \leq 1 \\
4, & 0.5<\mathrm{R}_{\mathrm{tb}} \leq .75
\end{array}\right.
$$

Thus the test for the significance of a pixel becomes

$$
\begin{aligned}
& S_{n}(\tau)= \begin{cases}1, \max _{(i, j)}\left\{\left|C_{i, j}\right|\right\} \geq \underset{\text { ming }\left(T, T_{a}\right)}{ }(21) \\
0, \quad \text { other wise }\end{cases} \\
& \text { Simplified } S_{n}(\tau)=\left\{\begin{array}{lr}
1, & \max _{(i, j)}\left\{\left|C_{i, j}\right|\right\} \geq T_{R_{t b}} \\
0, & \text { other wise }
\end{array}\right.
\end{aligned}
$$

Where $T_{\text {Rtb }}$ denotes the adaptive threshold for different target bit-rates. The reclassified significance Coefficient, which ranges between $\mathrm{T}$ and $(\mathrm{T}-\mathrm{f})$, is treated as the normal significance coefficient.

However, the remaining bits are set to zero. Physically, for a given target bit-rate, we can deduce the possible number of discarded bits. The majority of the quantization errors are hidden at different target bit-rates. The second modification does not reduce the coding performance. The first bit is the "significance" or "insignificance" bit, and the second bit is the sign bit. We can insert more where $\mathrm{T}_{\mathrm{Rtb}}$ denotes the adaptive threshold for different target bitrates. The reclassified significance coefficient, which ranges between $\mathrm{T}$ and $(\mathrm{T}-\mathrm{f})$, is treated as the normal significance coefficient. However, the remaining bits are set to zero. Physically, for a given target bit-rate, we can deduce the possible number of discarded bits. The majority of the quantization errors are hidden at different target bit-rates. The second modification does not reduce the coding performance. We can insert more zeros in the successive coding bit-planes to increase the coding efficiency in entropy coding, even if the first modification is not conducted.

\subsection{Simpler SPIHT Algorithm}

By using the given two above modifications, the simplified SPIHT encoding procedure, is as follows:

1. Initialization:

Obtain the target bit-rate and Output n; Set the LSP as an empty list, and add the coordinates $(i, j) \in H$. Add only the coordinates with descendants also to the LIS as type A entries.
2) Sorting Pass:

2.1) for each entry $(i, j)$ in the LIP:

2.1.1) output $S n(i, j)$;

2.1.2) If $\mathrm{Sn}(\mathrm{i}, \mathrm{j})=1$ by (22), then output the sign and $\mathrm{c}(\mathrm{i}$

$, j)\left(\mathrm{N}_{\mathrm{db}}\right)$ and delete $(\mathrm{i}, \mathrm{j})$ node.

2.2) for each entry $(i, j)$ in the LIS:

2.2.1) If the entry is of type A then

Output Sn (D (i, j));

If $\operatorname{Sn}(D(i, j))=1$ then

for each $(k, 1) \in O(i, j)$

Compute $\mathrm{Sn}(\mathrm{k}, \mathrm{l})$ by $(22)$;

If $\operatorname{Sn}(k, 1)=1$, then output the sign and

$\mathrm{c}(\mathrm{k}, \mathrm{l})\left(\mathrm{N}_{\mathrm{db}}\right)$ and delete $(\mathrm{i}, \mathrm{j})$ node;

If $\operatorname{Sn}(\mathrm{k}, 1)=0$, then add $(\mathrm{k}, 1)$ to the end of the LIP ;

If $\mathrm{L}(\mathrm{i}, \mathrm{j}) \neq \varphi$, then move $(\mathrm{i}, \mathrm{j})$ to the end of the LIS as an entry of type B; go to Step 2.2.2; otherwise, remove entry $(i, j)$ from the LIS.

2.2.2) If the entry is of type $B$, then

Output Sn (L (i, j));

If $\operatorname{Sn}(\mathrm{L}(\mathrm{i}, \mathrm{j}))=1$ then add each $(\mathrm{k}, \mathrm{l}) \in$

$\mathrm{O}(\mathrm{i}, \mathrm{j})$ to the end of the LIS as an entry of type A; Remove

$(i, j)$ from the LIS.

2.3) Threshold Update: decrease $n$ by 1 ;

2.4) go to step 2 .

\section{SIMULATION RESULTS}

The objective image quality metrics like Compression ratio, Peak Signal to Noise Ratio (PSNR), or Mean Squared Error (MSE) are best for the image processing application with bpp (bits per pixel).

Compression ratio: It also known as compression power is a term used to quantify the reduction in image representation size produced by a image compression algorithm. The data compression ratio is analogous to the physical compression ratio used to measure physical compression of substances, and is defined in the same way, as the ratio between the uncompressed image size and the compressed image size:

Compression Ratio=Uncompressed image Size/Compressed image Size.

Peak signal to noise ratio often abbreviated PSNR, is an engineering term for the ratio between the maximum possible power of a signal and the power of corrupting noise that affects the fidelity of its representation. Because many signals have a very wide dynamic range, PSNR is usually expressed in terms of the logarithmic decibel scale. The PSNR is most commonly used as a measure of quality of reconstruction in image compression etc. It is most easily defined via the mean squared error (MSE) defined as by averaging the squared intensity difference of reconstructed image, $\widehat{c}$ and the original image, c. Then from it the MSE is calculated as,

$\operatorname{MSE}=\frac{1}{M N}[c(i, j)-\widehat{c}(i, j)]^{2}$

where $\mathrm{c}(\mathrm{i}, \mathrm{j})$ is the original image, $\hat{\mathrm{c}}(\mathrm{i}, \mathrm{j})$ is the approximated version (which is actually the decompressed image) and M,N are the dimensions of the images Where, $\mathrm{MxN}$ is the size of the image and assuming the grey scale image of 8 bits per pixel (bpp), then the PSNR is defined as, 
$P S N R=10 \log _{10}\left[\frac{255^{2}}{M S E}\right]$

In this paper the performance of the discrete curvelet wrapped technique with simplified SPIHT is compared with that of the wavelet transform with original SPIHT using simulations. PSNR is computed from the mean square error, obtained from the difference of source and processed image, and defined in $\mathrm{dB}$. The tests are performed using the well-known "Lena" image with dimensions $256 \times 256$ pixels. The PSNR is measured at each bit-rate.

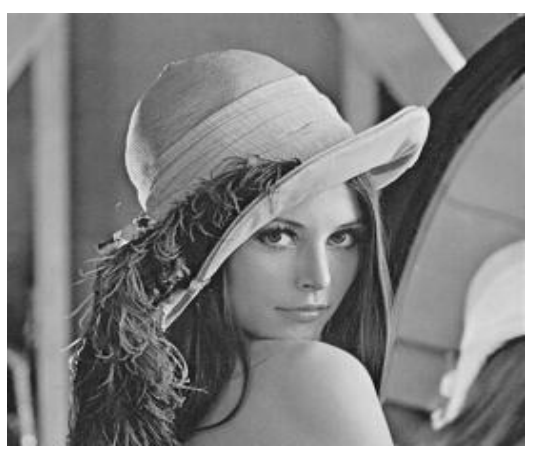

Original, 8bpp

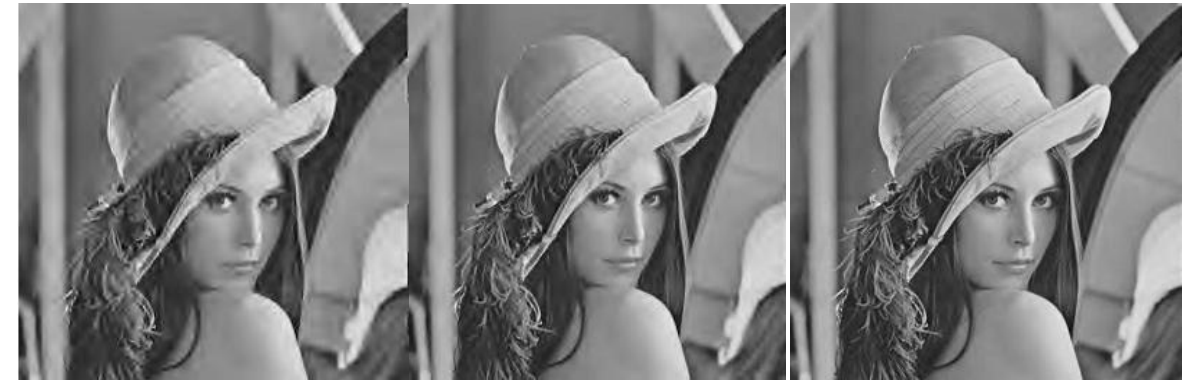

(a) $0.05 \mathrm{bpp}(122: 1)$

(b) $0.1 \mathrm{bpp}(61: 1)$

(c) $0.2 \mathrm{bpp}(45: 1)$

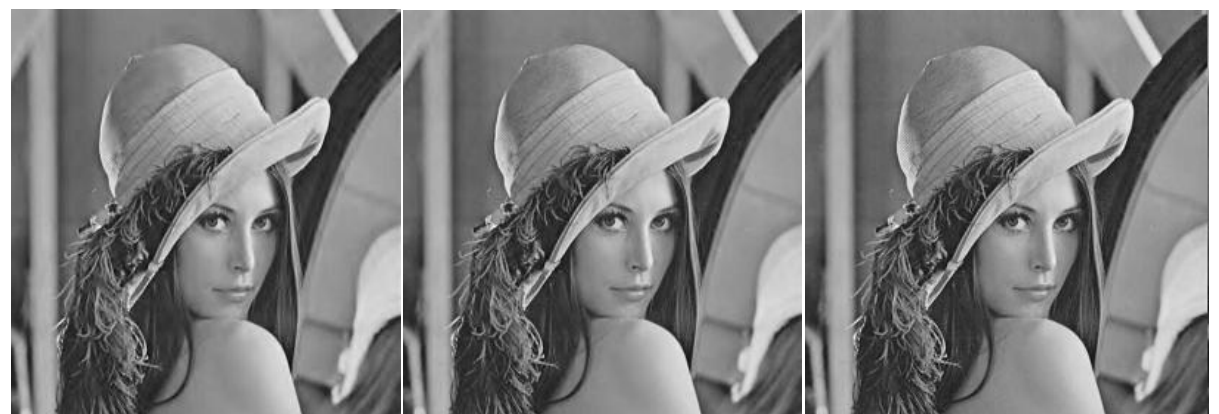

(d) $0.25 \mathrm{bpp}(32: 1)$

(e) $0.5 \mathrm{bpp}(15: 1)$

(f) $1.0 \mathrm{bpp}(7.6: 1)$

Figure 2: Lena image PSNR values: (a) 27.24dB. (b) 30.98dB. (c) 32.55dB. (d) 33.14dB. (e) 41.04dB. (f) $47.12 \mathrm{~dB}$ 
Table 1: Comparisons results of Lena

\begin{tabular}{|c|c|c|c|c|c|c|}
\hline \multirow[b]{2}{*}{$\begin{array}{l}\text { Bit-rate per } \\
\text { pixel(bpp) }\end{array}$} & \multicolumn{2}{|c|}{ CR(Compression Ratio) } & \multicolumn{2}{|c|}{$\begin{array}{l}\text { RMSE(Root Mean Square } \\
\text { Error) }\end{array}$} & \multicolumn{2}{|c|}{$\begin{array}{c}\text { PSNR(Peak Signal To Noise } \\
\text { Ratio) }\end{array}$} \\
\hline & $\begin{array}{c}\text { Proposed } \\
\text { scheme }\end{array}$ & $\begin{array}{c}\text { Wavelet With } \\
\text { SPHIT }\end{array}$ & $\begin{array}{l}\text { Proposed } \\
\text { scheme }\end{array}$ & $\begin{array}{c}\text { Wavelet With } \\
\text { SPHIT }\end{array}$ & $\begin{array}{c}\text { Proposed } \\
\text { scheme }\end{array}$ & $\begin{array}{c}\text { Wavelet With } \\
\text { SPHIT }\end{array}$ \\
\hline 0.005 & 124.2 & 96 & 12.50 & 14.68 & 27.24 & 24.74 \\
\hline 0.1 & 62.1 & 48 & 10.3 & 13.37 & 30.98 & 25.90 \\
\hline 0.2 & 45.1 & 36 & 9.49 & 12.94 & 32.55 & 27.11 \\
\hline 0.25 & 32.5 & 24 & 8.12 & 11.60 & 33.14 & 30.62 \\
\hline 0.5 & 15.3 & 12 & 7.10 & 10.68 & 41.04 & 36.04 \\
\hline 1 & 7.6 & 6 & 6.34 & 8.32 & 47.12 & 41.24 \\
\hline
\end{tabular}

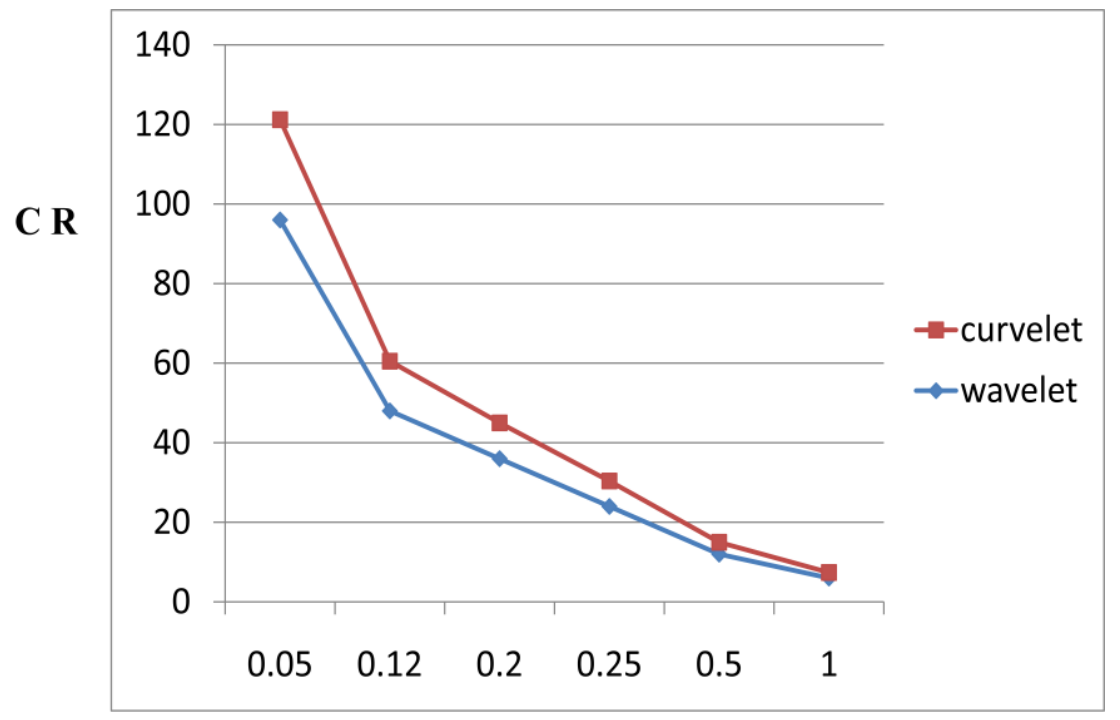

BIT RATE

Figure 3: comparison of wavelet and proposed algorithm Bit Rate with compression ratio 


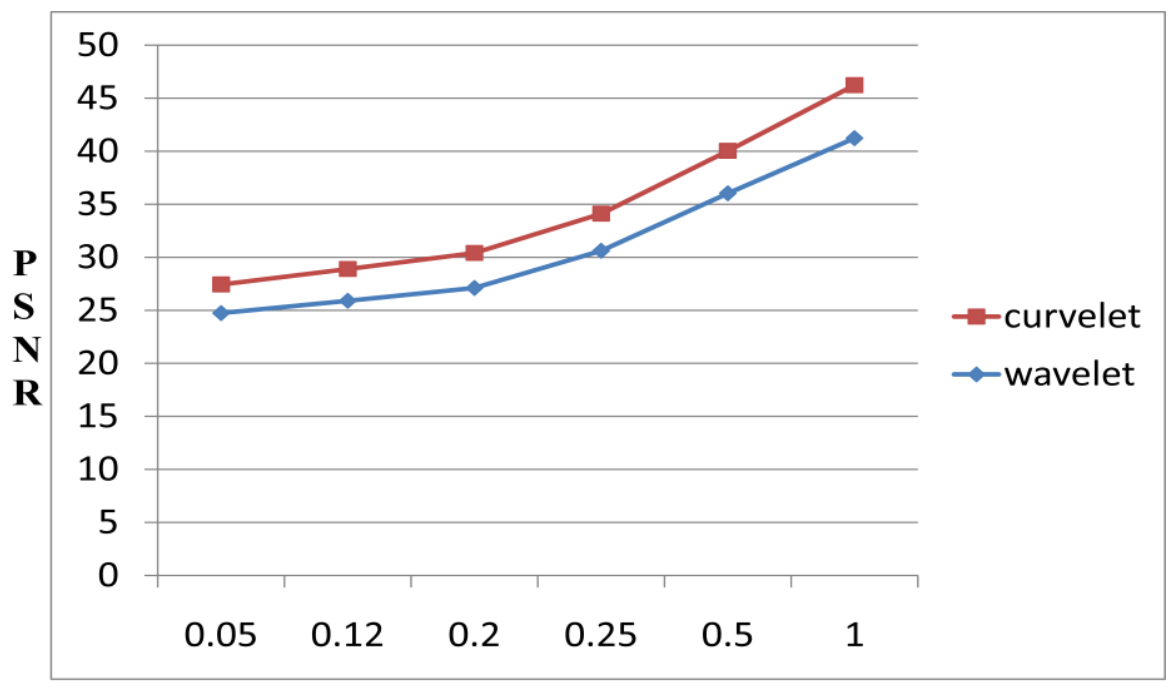

BIT RATE

Figure 4: comparison of wavelet and proposed algorithm Bit Rate with PSNR

\section{CONCLUSIONS}

This paper proposes new image compression technique using the curvelet wrapping technique with improved SPHIT allows for high compression ratios that conventional compression is unable to achieve. The algorithm is tested on varieties of benchmark images. Simulation results for one of the standard image, i.e., Lena with different BPP are presented. These results are compared with classical wavelet transform with SPHIT based image compression scheme. Experimental results clearly show that the proposed compression technique results in higher quality reconstructed images compared to that of other algorithms operating at similar bit rates for the class of images where edges are dominant with high compression ratio with good PSNR. The results are found to be better comparable with wavelet based compression with SPHIT which is more discontinuous with straight line singularity. According to the experimental results, our technique outperformed over the wavelet with SPHIT based image compression. It can be concluded that the integration of classical with soft computing based image compression methods enables a new way for achieving higher compression ratio. In future prospects of proposed methodology we are extending this work for video compression applications.

\section{REFERENCES}

[1] Y.-T. Chen and D.-C. Tseng, "Wavelet-based medical image compression with adaptive prediction, "Computerized Medical Imaging and Graphics, vol.31, Is.1, pp.1-8, Jan. 2007.

[2] V. N. Ramaswamy, K. R. Namuduri, and N. Ranganathan, "Context-based lossless image coding using EZW framework," IEEE Trans. Circuits, Syst., Video Technol., vol.11,pp.554-559, Apr. 2001.

[3] A. Said and W. A. Pearlman, "A new, fast, and efficient image codec based on set partitioning of hierarchical trees," IEEE Trans. Circuits, Syst., Video Technol., vol.6, pp.243-250, June 1996.

[4] C.Christopoulos, A. Skodras, and T. Ebrahimi, "JPEG2000 still image coding system: an overview,"
IEEE Trans. Consumer Electronics, vol.46, pp.11031127, Nov. 2000.

[5] G. Dillen, B. Georis, J. Legat, and O. Cantineau,"Combinedline-based architecture for the 5-3 and 9-7 wavelet transform of JPEG2000," IEEE Trans. Circuits and Systems for Video Technology, vol.13, pp.944-950, Sep. 2003.

[6] Z. Liu, Z. Xiong, Q. Wu, Y. Wang, and K. Castleman, "Cascaded differential and wavelet compression of chromosome images," IEEE Trans. Biomedical Engineering, vol. 49, pp.372-383, Apr. 2002.

[7] S. Tai, Y. Chen, and W. Yan, "New high-fidelity medical image compression based on modified set partitioning in hierarchical trees," Optical Engineering, vol.42, pp.1956-1963, July 2003.

[8] David L.Donoho and Mark R. Duncan, "Digital Curvelet Transform: Strategy, Implementation and Experiments" Nov 1999.

[9] Emmanuel Cand`es, Laurent Demanet, David Donoho and Lexing Ying. "Fast Discrete Curvelet Transform,”Jul 2005.

[10] A. Said and. A. Pearlman, "Image compression using Spatial-orientation tree," in Proc. IEEE Int. Symp. Circuits and Systems, Chicago, IL, May 1993, pp. 279-282.

[11] E.J. Candes, D.L. Donoho,"Curvelets A surprisingly effective nonadaptive representation for objects with edges", Curve and Surface Fitting, Vanderbilt Univ.Press 1999.

[12] E.J. Candes, D.L. Donahue,"New Tight Frames of Curvelets and Optimal Representations of Objects with Smooth Singularities", Technical Report, Stanford University, 2002.

[13] E.J. Candes, L. Demanet, D.L. Donoho , L. Ying,"Fast Discrete Curvelet Transforms" Technical Report, Cal Tech, 2005. 
[14] E. Candès and L. Demanet, "The curvelet representation of wave propagators is optimally sparse," Commun. Pure Appl. Math., vol. 58, no. 11, pp. 1472-1528, 2005

[15] A. Dutt and V. Rokhlin, Fast Fourier transforms for nonequispaced data II. Appl. Comput

[16] A. Said and W. Pearlman, "A New, fast and Efficient Image Codec based on Set Partitioning in Hierarchical Trees," IEEE Transactions on Circuits and Systems for Video technology, Vol. 6, No. 3, pp. 243 - 250, June 1996

[17] Jun Ren Ding and Jar Ferr Yang, "a simplified SPHIT algorithm", Journal of the Chinese Institute of Engineers, Vol. 31, No. 4, pp. 715-719 (2008).

\section{Siva Nagi Reddy.Kalli: Bio-Data}

SIVANAGIREDDY.KALLI received his B.E. degree in ECE the M.E. degree in VLSI DESIGN, and pursuing the Ph.D. in image processing algorithms implementing on FPGA. He had 9 years teaching experience .He was a, Assistant professor, with Department of ECE in repudiated institutions from 2003to2006 and Associate professor, in ARJUN College of Technology Science, HYDERABAD .At present working as Associate professor and In-charge Principal in MSIT-College of Engineering in Mylavaram, Vijayawada, A.P. He was published 5 International papers and his research interests include Digital image processing, VLSI Design, FPGA, Analog and digital communications. 